\title{
Convenient Stability Criteria for Difference Approximations of Hyperbolic Initial-Boundary Value Problems
}

\author{
By Moshe Goldberg* and Eitan Tadmor**
}

To Eugene Isaacson on his 65 th birthday

\begin{abstract}
New convenient stability criteria are provided in this paper for a large class of finite-difference approximations to initial-boundary value problems associated with the hyperbolic system $\mathbf{u}_{t}=A \mathbf{u}_{x}+B \mathbf{u}+\mathbf{f}$ in the quarter plane $x \geqslant 0, t \geqslant 0$. Using the new criteria, stability is easily established for numerous combinations of well-known basic schemes and boundary conditions, thus generalizing many special cases studied in the recent literature.
\end{abstract}

0. Introduction. In this paper we extend the results of [3] to obtain convenient, more versatile, sufficient stability criteria for a wide class of difference approximations for initial-boundary value problems associated with the hyperbolic system $\mathbf{u}_{t}=A \mathbf{u}_{x}+B \mathbf{u}+\mathbf{f}$ in the quarter plane $x \geqslant 0, t \geqslant 0$. Our difference approximation consists of a general difference scheme-explicit or implicit, dissipative or not, two-level or multi-level—and boundary conditions of the type discussed in [3].

We restrict attention to the case where the outflow part of our boundary conditions is translatory; i.e., determined at all boundary points by the same coefficients. Such boundary conditions are commonly used in practice; and in particular, when the boundary consists of a single point, the boundary conditions are translatory by definition.

Throughout the paper we assume that the basic scheme is stable for the pure Cauchy problem, and that the assumptions that guarantee the validity of the stability theory of Gustafsson, Kreiss and Sundström [5] hold in our case. With this in mind, we raise the question of stability for our approximation in the sense of Definition 3.3 in [5].

We begin our stability analysis in Secton 1 by recalling Theorem 2.1 of [3], which implies that the entire approximation is stable if and only if the scalar outflow

\footnotetext{
Received September 26, 1983.

1980 Mathematics Subject Classification. Primary 65M10; Secondary 65N10.

${ }^{*}$ Research sponsored in part by the Air Force Office of Scientific Research, Air Force System Command, United States Air Force Grant Nos. AFOSR-79-0127 and AFOSR-83-0150. Part of this research was carried out while the author visited the Institute for Computer Applications in Science and Engineering, NASA Langley Research Center, Hampton, VA 23665.

**Research sponsored in part by the National Aeronautical and Space Administration under NASA Contract No. NASA-17070 while the author was in residence at the Institute for Computer Applications in Science and Engineering, NASA Langley Research Center, Hampton, VA 23665.
} 
components of its principal part are stable. Thus, our global stability question is reduced to that of a scalar, homogeneous, outflow problem which is the main subject of this paper.

Our stability criteria for the reduced problem - stated in the first part of Section 2 and proven in Section 3-depend both on the basic scheme and the boundary conditions, but very little on the intricate interaction between the two. Consequently, our new criteria provide in many cases a convenient alternative to the well-known Gustafsson-Kreiss-Sundström criterion in [5].

In the second part of Section 2, we use the new stability criteria to reestablish all the main examples in our previous paper [3]. We show that if the basic scheme is arbitrary (dissipative or not) and the boundary conditions are generated by either the explicit or implicit right-sided Euler schemes, then overall stability is assured. For dissipative basic schemes we prove stability if the boundary conditions are determined by either oblique extrapolation, by the Box-Scheme, or by the right-sided weighted Euler scheme.

Section 2 contains some new examples as well. Among these we find that if the basic scheme is arbitrary and two-level, then horizontal extrapolation at the boundary maintains overall stability. Other stable cases occur when the basic scheme is given by either the Crank-Nicolson scheme or by the backward (implicit) Euler scheme, and the boundary conditions are determined by oblique extrapolation. Such examples, where neither the basic scheme nor the boundary conditions are dissipative, could not have been handled by our previous results in [3].

All told, our examples in this paper incorporate most of the cases discussed in the recent literature; e.g. [1]-[3], [5], [6], [8]-[10], [12], [13], [15], [16].

As in [3], we point out that there is no difficulty in extending our stability criteria to problems with two boundaries. This is so since, if the corresponding left and right quarter-plane problems are stable, then by Theorem 5.4 of [5], the original problem is stable as well.

1. The Difference Approximation and the Reduced Problem. Consider the first-order hyperbolic system of partial differential equations

(1.1a) $\partial \mathbf{u}(x, t) / \partial t=A \partial \mathbf{u}(x, t) / \partial x+B \mathbf{u}(x, t)+\mathbf{f}(x, t), \quad x \geqslant 0, t \geqslant 0$, where $\mathbf{u}(x, t)=\left(u^{(1)}(x, t), \ldots, u^{(n)}(x, t)\right)^{\prime}$ is the unknown vector (prime denoting the transpose), $\mathbf{f}(x, t)=\left(f^{(1)}(x, t), \ldots, f^{(n)}(x, t)\right)^{\prime}$ is a given vector, and $A$ and $B$ are fixed $n \times n$ matrices such that $A$ is Hermitian and nonsingular. Without restriction we may assume that $A$ is diagonal of the form

$$
A=\left(\begin{array}{cc}
A^{\mathrm{I}} & 0 \\
0 & A^{\mathrm{II}}
\end{array}\right), \quad A^{\mathrm{I}}<0, A^{\mathrm{II}}>0,
$$

where $A^{\mathrm{I}}$ and $A^{\mathrm{II}}$ are of orders $l \times l$ and $(n-l) \times(n-l)$, respectively.

The solution of (1.1) is determined uniquely if we prescribe initial values

$$
\mathbf{u}(x, 0)=\mathbf{u}^{0}(x), \quad x \geqslant 0
$$

and boundary conditions

$$
\mathbf{u}^{\mathrm{I}}(0, t)=S \mathbf{u}^{\mathrm{II}}(0, t)+\mathbf{g}(t), \quad t \geqslant 0
$$


where $S$ is a fixed $l \times(n-l)$ matrix, $\mathbf{g}(t)$ is a given $l$-vector, and

$$
\mathbf{u}^{\mathrm{I}}=\left(u^{(1)}, \ldots, u^{(l)}\right)^{\prime}, \quad \mathbf{u}^{\mathrm{II}}=\left(u^{(l+1)}, \ldots, u^{(n)}\right)^{\prime}
$$

is a partition of $\mathbf{u}$ into its inflow and outflow components, corresponding to the partition of $A$ in (1.2).

In order to solve the initial-boundary value problem (1.1) by a finite-difference approximation we introduce, as usual, a mesh size $\Delta x>0, \Delta t>0$, such that $\lambda \equiv \Delta t / \Delta x=$ constant; and using the notation $\mathbf{v}_{\nu}(t)=\mathbf{v}(\nu \Delta x, t)$, we approximate (1.1a) by a general, consistent, two-sided, solvable, multi-level basic scheme of the form

$$
\begin{gathered}
Q_{-1} \mathbf{v}_{\nu}(t+\Delta t)=\sum_{\sigma=0}^{s} Q_{\sigma} \mathbf{v}_{\nu}(t-\sigma \Delta t)+\Delta t \mathbf{b}_{\nu}(t), \\
Q_{\sigma}=\sum_{j=-r}^{p} A_{j \sigma} E^{j}, \quad E \mathbf{v}_{\nu}=\mathbf{v}_{\nu+1}, \quad \sigma=-1, \ldots, s, \nu=r, r+1, \ldots
\end{gathered}
$$

Here, the $n \times n$ matrices $A_{j \sigma}$ are polynomials in $A$ and $\Delta t B$, and the $n$-vectors $\mathbf{b}_{\nu}(t)$ depend smoothly on $\mathbf{f}(x, t)$ and its derivatives.

The equations in (1.4a) have a unique solution if we provide initial values

$$
\mathbf{v}_{\nu}(\sigma \Delta t)=\mathbf{v}_{\nu}^{0}(\sigma \Delta t), \quad \sigma=0, \ldots, s, \nu=0,1,2, \ldots,
$$

where in addition we must specify, at each time step $t=\mu \Delta t, \mu=s, s+1, \ldots$, boundary values $\mathbf{v}_{\nu}(t+\Delta t), \nu=0, \ldots, r-1$. As in [3], these boundary values will be determined by two sets of boundary conditions, the first of which is obtained by taking the last $n-l$ components of general boundary conditions of the form

$$
\begin{gathered}
T_{-1} \mathbf{v}(t+\Delta t)=\sum_{\sigma=0}^{q} T_{\sigma} \mathbf{v}_{\nu}(t-\sigma \Delta t)+\Delta t \mathbf{d}_{\nu}(t), \\
T_{\sigma}=\sum_{j=0}^{m} C_{j \sigma} E^{j}, \quad \sigma=-1, \ldots, q, \nu=0, \ldots, r-1,
\end{gathered}
$$

where the matrices $C_{j \sigma}$ are polynomials in $A$ and $\Delta t B$, the $C_{j(-1)}$ are nonsingular, and the $n$-vectors $\mathbf{d}_{\nu}(t)$ are functions of $\mathbf{f}(x, t)$ and its derivatives.

If we put

$$
C_{j \sigma}=\left(\begin{array}{cc}
C_{j \sigma}^{\mathrm{II}} & C_{j \sigma}^{\mathrm{II}} \\
C_{j \sigma}^{\mathrm{II}} & C_{j \sigma}^{\mathrm{II} \mathrm{II}}
\end{array}\right), \quad \mathbf{v}_{\nu}=\left(\begin{array}{c}
\mathbf{v}_{\nu}^{\mathrm{I}} \\
\mathbf{v}_{\nu}^{\mathrm{II}}
\end{array}\right), \quad \mathbf{d}_{\nu}=\left(\begin{array}{c}
\mathbf{d}_{\nu}^{\mathrm{I}} \\
\mathbf{d}_{\nu}^{\mathrm{II}}
\end{array}\right)
$$

in accordance with the partition of $A$ and $\mathbf{u}$ in (1.2), (1.3), then this set of conditions takes the explicit form

$$
\begin{aligned}
& T_{-1}^{\mathrm{II} \mathrm{I}} \mathbf{v}_{\nu}^{\mathrm{I}}(t+\Delta t)+T_{-1}^{\mathrm{II} \mathrm{II}} \mathbf{v}_{\nu}^{\mathrm{II}}(t+\Delta t) \\
& =\sum_{\sigma=0}^{q}\left[T_{\boldsymbol{\sigma}}^{\mathrm{II} \mathrm{I}} \mathbf{v}_{\nu}^{\mathrm{I}}(t-\sigma \Delta t)+T_{\boldsymbol{\sigma}}^{\mathrm{II} \mathrm{II}} \mathbf{v}_{\nu}^{\mathrm{II}}(t-\sigma \Delta t)\right]+\Delta t \mathbf{d}_{\nu}^{\mathrm{II}}(t), \\
& T_{\boldsymbol{\sigma}}^{\mathrm{II} \alpha}=\sum_{j=0}^{m} C_{\boldsymbol{\sigma}}^{\mathrm{II} \alpha} E^{j}, \quad \alpha=\mathrm{I}, \mathrm{II}, \nu=0, \ldots, r-1 .
\end{aligned}
$$


We call such boundary conditions translatory since they are determined at all boundary points by the same coefficients.

For the second set of boundary conditions we use the analytic condition

$$
\mathbf{v}_{0}^{\mathrm{I}}(t+\Delta t)=S \mathbf{v}_{0}^{\mathrm{II}}(t+\Delta t)+\mathbf{g}(t+\Delta t),
$$

together with $r-1$ additional conditions (not necessarily translatory) of the form

$$
\begin{aligned}
\mathbf{v}_{\nu}^{\mathrm{I}}(t+\Delta t)=\sum_{j=1}^{k}\left[D_{j \nu}^{\mathrm{II}} \mathbf{v}_{j}^{\mathrm{I}}(t+\Delta t)+D_{j \nu}^{\mathrm{III}} \mathbf{v}_{j}^{\mathrm{II}}(t+\Delta t)\right]+\Delta t \mathbf{e}_{\nu}^{\mathrm{I}}(t), & \\
& \nu=1, \ldots, r-1,
\end{aligned}
$$

where the matrices $D_{j \nu}^{\mathrm{II}}$ and $D_{j \nu}^{\mathrm{II}}$ - of orders $l \times l$ and $l \times(n-l)$ respectively-are polynomials in the blocks $A^{\alpha}$ and $\Delta t B^{\alpha \beta}, \alpha, \beta=\mathrm{I}$, II, of the matching partitions

$$
A=\left(\begin{array}{cc}
A^{\mathrm{I}} & 0 \\
0 & A^{\mathrm{II}}
\end{array}\right), \quad \Delta t B=\Delta t\left(\begin{array}{cc}
B^{\mathrm{II}} & B^{\mathrm{III}} \\
B^{\mathrm{II} \mathrm{I}} & B^{\text {II II }}
\end{array}\right),
$$

so that the $D_{j \nu}^{\mathrm{I} I}$ vanish whenever $B$ does; and the $l$-vectors $\mathbf{e}_{\nu}^{\mathrm{I}}(t)$ are functions of $\mathbf{f}(x, t), \mathbf{g}(t)$, and their derivatives.

It has been shown in [3] that Eqs. (1.4c)-(1.4e) can be solved uniquely for the required boundary values $\mathbf{v}_{\nu}(t+\Delta t), \nu=0, \ldots, r-1$, in terms of neighboring values of $\mathbf{v}$, at least for sufficiently small $\Delta t$; and that boundary conditions of the form (1.4e) can be constructed to any degree of accuracy. A concrete example of second-order accurate boundary conditions of the form (1.4e), for the special case $B=\mathbf{f}=0$, was given in [2].

The difference approximation is completely defined now by (1.4); so assuming that the basic scheme is stable for the pure Cauchy problem $(-\infty<\nu<\infty)$, we may ask whether the entire approximation is stable. More precisely, we make from now on the same assumptions about approximation (1.4) as in [5], so that the stability theory of Gustafsson, Kreiss and Sundström holds, and we raise the above stability question in the sense of Definition 3.3 of [5].

As in [3], the first step will be to reduce our stability question to that of a scalar, outflow approximation with homogeneous translatory boundary conditions. This reduction is obtained by applying $(1.4 \mathrm{a})-(1.4 \mathrm{c})$ to the scalar outflow problem

$$
u_{t}=a u_{x}, a=\text { constant }>0, \quad x \geqslant 0, t \geqslant 0
$$

(which requires no analytic boundary conditions), where $a$ varies over the eigenvalues of $A^{\text {II }}$. In other words, we set

$$
A^{\mathrm{I}}=B=\mathbf{f}=0, \quad A^{\mathrm{II}}=a=\text { constant }>0 ;
$$

so that (1.1a) yields $(1.5)$; and $(1.4 \mathrm{a})-(1.4 \mathrm{c})$ reduces to a self-contained, scalar, homogeneous approximation which consists of the basic scheme

$$
\begin{aligned}
Q_{-1} v_{\nu}(t+\Delta t) & =\sum_{\sigma=0}^{s} Q_{\sigma} v_{\nu}(t-\sigma \Delta t), \quad \nu=r, r+1, \ldots, \\
Q_{\sigma} & =\sum_{j=-r}^{p} a_{j \sigma} E^{j}, \quad \sigma=-1, \ldots, s
\end{aligned}
$$

with initial values

$$
v_{\nu}(\sigma \Delta t)=v_{\nu}^{0}(\sigma \Delta t), \quad \sigma=0, \ldots, s, \nu=0,1,2, \ldots ;
$$


and translatory outflow boundary conditions

$$
\begin{gathered}
T_{-1} v_{\nu}(t+\Delta t)=\sum_{\sigma=0}^{q} T_{\sigma} v_{\nu}(t-\sigma \Delta t), \quad \nu=0, \ldots, r-1, \\
T_{\sigma}=\sum_{j=0}^{m} c_{j \sigma} E^{j}, \quad c_{0(-1)} \neq 0, \quad \sigma=-1, \ldots, q,
\end{gathered}
$$

where the scalars $a_{j \sigma}$ and $c_{j \sigma}$ are polynomials in $a$, and the basic scheme (1.6a) is consistent with (1.5).

We are now ready to state our main result in Section 2 of [3], which we reformulate as follows:

TheOREM 1.1 ([3, Theorem 2.1]). Approximation (1.4) is stable if and only if its reduced form in (1.6) is stable for every eigenvalue a of $A^{\mathrm{II}}$. That is, approximation (1.4) is stable if and only if the scalar outflow components of its principal part are stable.

The above reduction theorem implies that from now on we may restrict our stability study to the scalar outflow approximation (1.6). Hence, we conclude this section by stating the following five assumptions which will hold throughout the paper, and guarantee the validity of the Gustafsson-Kreiss-Sundström theory [5] for approximation (1.6).

Assumption 1.1 ([5, Assumption 3.1]; [3, Assumption 2.1]). Approximation (1.6) is boundedly solvable; i.e., there exists a constant $K>0$ such that for each $y \in l_{2}(\Delta x)$ there is a unique solution $w \in l_{2}(\Delta x)$ to the equations

$$
\begin{aligned}
Q_{-1} w_{\nu}=y_{\nu}, & \nu=r, r+1, \ldots, \\
T_{-1} w_{\nu}=y_{\nu}, & \nu=0, \ldots, r-1,
\end{aligned}
$$

with $\|w\| \leqslant K\|y\|$; where $Q_{-1}$ and $T_{-1}$ are defined in (1.6a, c), and $l_{2}(\Delta x)$ is the space of all grid functions $w=\left\{w_{\nu}\right\}_{\nu=0}^{\infty}$ with $\|w\|^{2} \equiv \Delta x \sum_{\nu=0}^{\infty}\left|w_{\nu}\right|^{2}<\infty$.

Assumption 1.2 ([5, Assumption 5.1]; [3, Assumption 2.2]). The basic scheme is stable for the pure Cauchy problem $-\infty<\nu<\infty$. That is, if we define the basic characteristic function by

$$
P(z, \kappa)=\sum_{j=-r}^{p} a_{j}(z) \kappa^{j}
$$

where

$$
a_{j}(z) \equiv a_{j(-1)}-\sum_{\sigma=0}^{s} z^{-\sigma-1} a_{j \sigma}, \quad j=-r, \ldots, p
$$

then we have:

(i) The von Neumann condition; i.e., the solutions $z(\kappa)$ of the basic characteristic equation

$$
P(z, \kappa)=0
$$

satisfy

$$
|z(\kappa)| \leqslant 1 \quad \text { for all } \kappa \text { with }|\kappa|=1 .
$$

(ii) If $|\kappa|=1$, and if $z(\kappa)$ is a root of (1.9) with $|z(\kappa)|=1$, then $z(\kappa)$ is a simple root of (1.9). 
Assumption 1.3 (see [5, Assumption 5.4 together with Definition 10.1]; compare [3, Assumption 2.3], and Osher [10]). The basic scheme (1.6a) belongs to the family of schemes for which the Gustafsson-Kreiss-Sundström theory in [5] holds. This family contains in particular the following two classes:

(i) Dissipative basic schemes; i.e., schemes for which the roots $z(\kappa)$ of (1.9) satisfy

$$
|z(\kappa)|<1 \quad \text { for all } \kappa \text { with }|\kappa|=1, \kappa \neq 1 .
$$

(ii) Unitary basic schemes (also known as strictly nondissipative schemes) where the roots of (1.9) satisfy

$$
|z(\kappa)|=1 \text { for all }|\kappa|=1 .
$$

Obviously, if the basic scheme belongs to any of these two classes, then it satisfies the von Neumann condition in Assumption 1.2(i).

Assumption 1.4 ([5, Assumption 5.5]; [3, Assumption 2.4]).

$$
a_{-r}(z), a_{p}(z) \neq 0 \text { for all }|z| \geqslant 1 \text {. }
$$

Assumption 1.5. We assume that

$$
\sum_{j=0}^{m}\left|c_{j}(z)\right| \neq 0 \text { for all }|z| \geqslant 1
$$

where, in analogy with (1.8),

$$
c_{j}(z) \equiv c_{j(-1)}-\sum_{\sigma=0}^{q} z^{-\sigma-1} c_{j \sigma}, \quad j=0, \ldots, m .
$$

Assumption 1.5 is necessary for stability, as shown in Remark 3.4 below. This last assumption - which should have been included in [3] as well-is easily verified for all practical boundary conditions.

2. Statement of Results and Examples. In order to state our main stability criteria we define, in complete analogy with (1.7), the boundary characteristic function

$$
R(z, \kappa)=\sum_{j=0}^{m} c_{j}(z) \kappa^{j}
$$

where the $c_{j}(z)$ are given in (1.12). Defining the function

$$
\Omega(z, \kappa) \equiv|P(z, \kappa)|+|R(z, \kappa)|,
$$

we shall prove

THEOREM 2.1 (1ST MAIN THEOREM). Approximation (1.6) is stable if

$$
\Omega(z, \kappa) \neq 0 \text { for all }|z| \geqslant 1,0<|\kappa| \leqslant 1,(z, \kappa) \neq(1,1) .
$$

Next, let us divide the $(z, \kappa)$ domain in $(2.1)$ into three disjoint parts and restate Theorem 2.1 as follows:

TheOrem 2.1' (1ST MAIN TheOREM REVISITED). Approximation (1.6) is stable if

$$
\begin{array}{ll}
\Omega(z, \kappa) \neq 0 & \text { for all }|z| \geqslant 1,|\kappa|=1, \kappa \neq 1 \\
\Omega(z, \kappa=1) \neq 0 & \text { for all }|z| \geqslant 1, z \neq 1 \\
\Omega(z, \kappa) \neq 0 & \text { for all }|z| \geqslant 1,0<|\kappa|<1
\end{array}
$$


The advantage of this new setting is explained by Theorem 2.2 in which we provide useful sufficient conditions for each of the three inequalities in (2.2) to hold. Before stating this theorem, we need the following definitions:

Definition 2.1. The boundary conditions (1.6c) are said to be dissipative if the roots $z(\kappa)$ of the boundary characteristic equation

$$
R(z, \kappa)=0
$$

satisfy

$$
|z(\kappa)|<1 \text { for all }|\kappa| \leqslant 1, \kappa \neq 1 .
$$

Definition 2.2. We say that the boundary conditions (1.6c) satisfy the von Neumann condition if the roots $z(\kappa)$ of (2.3) satisfy

$$
|z(\kappa)| \leqslant 1 \text { for all }|\kappa|=1 \text {. }
$$

Definition 2.3. The boundary conditions (1.6c) are called boundedly solvable if there exists a constant $K>0$ so that for each $y \in l_{2}(\Delta x)$ there is a unique solution $w \in l_{2}(\Delta x)$ to

$$
T_{-1} w_{\nu}=y_{\nu}, \quad \nu=0,1,2, \ldots
$$

with $\|w\| \leqslant K\|y\|$.

Evidently, these definitions are analogous to those made for the basic scheme in Assumptions 1.3(i), 1.2(i) and 1.1, respectively; and again, dissipativity implies the von Neumann condition.

Having these definitions, we shall prove

ThEOREM 2.2 (2ND MAIN THEOREM). (i) If either the basic scheme or the boundary conditions are dissipative, then (2.2a) holds.

(ii) Inequality (2.2b) is satisfied if any one of the following holds:

(a) The basic scheme is two-level.

(b) The basic scheme is three-level and

$$
\Omega(z=-1, \kappa=1) \neq 0 .
$$

(c) The boundary conditions are two-level and at least of zero order of accuracy.

(d) The boundary conditions are three-level, at least zero-order accurate, and (2.5) is satisfied.

(iii) If the boundary conditions satisfy the von Neumann condition and are boundedly solvable, then (2.2c) holds.

The proofs of Theorems 2.1 and 2.2 are given in Section 3.

We turn now to examples. Before doing so, let us recall one more result which provides a useful sufficient condition for the boundary conditions to be boundedly solvable.

LEMMA 2.1 ([3, Lemma 3.2]). (i) The boundary conditions (1.6c) are boundedly solvable if and only if

$$
T_{-1}(\kappa) \equiv \sum_{j=0}^{q} c_{j(-1)} \kappa^{j} \neq 0 \quad \text { for all } 0<|\kappa| \leqslant 1 .
$$

(ii) In particular, explicit boundary conditions are always boundedly solvable. 
This result is associated with important observations on solvability by Osher [11].

We remark that part (i) of Lemma 3.2 in [3] merely states that if (2.6) holds, then the boundary conditions are boundedly solvable. Following the proof of Lemma 3.2 in [3], however, one realizes without difficulty that in fact, (2.6) is both sufficient and necessary for the bounded solvability of the boundary conditions (1.6c), as stated above.

Example 2.1 ([3, Example 3.5]; compare the special cases in [5, Theorems 6.1-6.3], [9, Theorems 18.1, 18.2], [12, (3.2)], and [2, Example 2]). Let the basic scheme (1.6a) be arbitrary (dissipative or not), and let the boundary conditions (1.6c) be generated by the right-sided, first-order accurate, explicit Euler scheme

$$
v_{\nu}(t+\Delta t)=v_{\nu}(t)+\lambda a\left[v_{\nu+1}(t)-v_{\nu}(t)\right], \quad 0<\lambda a<1, \nu=0, \ldots, r-1 .
$$

These two-level boundary conditions are known to be dissipative (see [3, Example $3.5]$ ), hence Theorem 2.2(i)(iic) implies $(2.2 \mathrm{a}, \mathrm{b})$. Since the boundary conditions are explicit, then by Lemma 2.1 they are solvable; and since they are dissipative, the von Neumann condition is satisfied. Consequently, Theorem 2.2(iii) yields (2.2c), and stability is established by Theorem $1.2^{\prime}$.

It is a trivial matter to verify that for this, as well as for all the following examples, Assumption 1.5 is fulfilled.

Example 2.2 ([3, Example 3.6]; compare the special cases in [12, (3.3)], and [2, Example 3]). Take an arbitrary basic scheme, and determine the boundary conditions by the right-sided, first-order accurate, implict Euler scheme

$$
v_{\nu}(t+\Delta t)-\lambda a\left[v_{\nu+1}(t+\Delta t)-v_{\nu}(t+\Delta t)\right]=v_{\nu}, \quad \lambda a>0, \nu=0, \ldots, r-1 .
$$

As in the previous example, the boundary conditions are two-level and dissipative (e.g. [3, Example 3.6]), so Theorem 2.2(i)(iic) gives (2.2a, b). The dissipativity of the boundary conditions also implies the von Neumann condition. And it is trivially verified ([3, Example 3.6] again) that $T_{-1}(\kappa) \neq 0$ for $|\kappa| \leqslant 1$ (where $T_{-1}(\kappa)$ is defined in (2.6)), so that Lemma 2.1(i) implies solvability. Thus, (2.2c) follows from Theorem 2.2(iii); and Theorem 2.1' assures stability.

Example 2.3 (compare the special cases in [8, Theorem 6], [10, Section XXIII], [5, Theorems 6.1 and 6.3], [1], [6, Theorem 2.1], and [3, Example 3.1]). Take an arbitrary two-level basic scheme, and define the boundary conditions by horizontal extrapolation of order $\omega-1$ :

$$
v_{\nu}(t+\Delta t)=\sum_{j=1}^{\omega}\left(\begin{array}{c}
\omega \\
j
\end{array}\right)(-1)^{j+1} v_{\nu+j}(t+\Delta t), \quad \nu=0, \ldots, r-1 .
$$

We have

$$
R(z, \kappa)=R(\kappa)=1-\sum_{j=1}^{\omega}\left(\begin{array}{l}
\omega \\
j
\end{array}\right)(-1)^{j+1} \kappa^{j}=(1-\kappa)^{\omega},
$$

so $R(\kappa) \neq 0$ for $\kappa \neq 1$, which directly gives $(2.2 \mathrm{a}, \mathrm{c})$. Moreover, since the basic scheme is two-level, Theorem 2.2(iia) implies (2.2b); and Theorem 2.1' again proves stability.

It is interesting to note that the above result may fail, both for nondissipative and dissipative basic schemes, if the basic scheme is of more than two time-levels. A nondissipative example was given in Theorem 6.2 of [5] by Gustafsson et al. who 
showed that the unitary, 3-level Leap-Frog scheme

$$
v_{\nu}(t+\Delta t)=v_{\nu}(t-\Delta t)+\lambda a\left[v_{\nu+1}(t)-v_{\nu-1}(t)\right], \quad \nu=1,2,3, \ldots,
$$

provides an unstable approximation in combination with the linear boundary extrapolation $((2.7)$ with $\omega=2)$ :

$$
v_{0}(t+\Delta t)=2 v_{1}(t+\Delta t)-v_{2}(t+\Delta t)
$$

We proved instability $([3,(3.6)])$ for the case where the basic scheme is the 3-level, 5-point, dissipative version of (2.8):

$$
\begin{array}{r}
v_{\nu}(t+\Delta t)=\left[I-\frac{\varepsilon}{16}(E-I)^{2}\left(I-E^{-1}\right)^{2}\right] v_{\nu}(t-\Delta t)+\lambda a\left(E-E^{-1}\right) v_{\nu}(t), \\
0<\varepsilon<1,0<\lambda a \leqslant 1-\varepsilon, \nu=2,3, \ldots,
\end{array}
$$

and the boundary conditions are given by (2.7) with $\nu=0,1$.

Example 2.4 ([3, Example 3.2]; compare the special cases in [2, Example 1] and [6, Theorem 2.2]). Let the basic scheme be dissipative, and determine the boundary conditions by oblique extrapolation of order $\omega-1$ :

$$
v_{\nu}(t+\Delta t)=\sum_{j=1}^{\omega}\left(\begin{array}{c}
\omega \\
j
\end{array}\right)(-1)^{j+1} v_{\nu+j}[t-(j-1) \Delta t], \quad \nu=0, \ldots, r-1
$$

Since the basic scheme is dissipative, then Theorem 2.2(i) implies (2.2a). Furthermore, the boundary characteristic function for (2.9) is

$$
R(z, \kappa)=1-\sum_{j=1}^{\omega}\left(\begin{array}{c}
\omega \\
j
\end{array}\right)(-1)^{j+1} z^{-j} \kappa^{j}=\left(1-z^{-1} \kappa\right)^{\omega}
$$

hence

$$
\Omega(z, \kappa) \geqslant|R(z, \kappa)| \neq 0, \quad z \neq \kappa .
$$

This yields $(2.2 \mathrm{~b}, \mathrm{c})$; and Theorem $2.1^{\prime}$ implies stability.

Example 2.5 ([3, Example 3.3]; compare the special cases [5, Theorem 6.1], [12, (3.4)], and [2, Example 4]). Take any dissipative basic scheme, and let the boundary conditions be generated by the second-order accurate Box-Scheme

$$
\begin{aligned}
v_{\nu}(t & +\Delta t)+v_{\nu+1}(t+\Delta t)-\lambda a\left[v_{\nu+1}(t+\Delta t)-v_{\nu}(t+\Delta t)\right] \\
& =v_{\nu}(t)+v_{\nu+1}(t)+\lambda a\left[v_{\nu+1}(t)-v_{\nu}(t)\right], \quad \nu=0, \ldots, r-1 .
\end{aligned}
$$

As in the previous example, dissipativity implies (2.2a); and Theorem 2.2(iic) implies (2.2b). Next, we recall Example 3.3 of [3] where it was shown without difficulty that the roots $z(\kappa)$ of the boundary characteristic function satisfy $|z(\kappa)|=1$ for $|\kappa|=1$, and that $T_{-1}(\kappa) \neq 0$ for $|\kappa| \leqslant 1$. Thus, the boundary conditions satisfy the von Neumann condition; and Lemma 2.1(i) implies solvability. With this, Theorem 2.2(iii) gives (2.2c), and by Theorem $2.1^{\prime}$ stability follows.

Example 2.6 ([3, Example 3.4]; compare the special case in [9, Theorem 18.1]). Take any dissipative basic scheme, and define the boundary conditions by the right-sided, 3-level, weighted Euler scheme

$$
\begin{aligned}
v_{\nu}(t+\Delta t)=v_{\nu}(t-\Delta t)+\lambda a\left[2 v_{\nu+1}(t)-v_{\nu}(t\right. & \left.+\Delta t)-v_{\nu}(t-\Delta t)\right] \\
0 & <\lambda a \leqslant 1, \nu=0, \ldots, r-1 .
\end{aligned}
$$

As in the two previous examples, Theorem 2.2(i) implies (2.2a). Further, we have

$$
R(z, \kappa)=1-z^{-2}-\lambda a\left(2 \kappa z^{-1}-1-z^{-2}\right) ;
$$


so

$$
\Omega(z=-1, \kappa=1) \geqslant|R(z=-1, \kappa=1)|=4 \lambda a>0,
$$

and Theorem 2.2(iid) yields (2.2b). Next, as in Example 3.4 of [3], we find that the roots of $R(z, \kappa)$ satisfy

$$
z\left(\kappa=e^{i \xi}\right)=e^{i \xi} \frac{\lambda a \pm b(\xi)}{\lambda a+1}, \quad b(\xi) \equiv \sqrt{(\lambda a)^{2}+e^{-2 i \xi}\left[1-(\lambda a)^{2}\right]}
$$

and since $0<\lambda a \leqslant 1$, then $|b(\xi)| \leqslant 1$ for $|\xi| \leqslant \pi$. Therefore

$$
\left|z\left(\kappa=e^{i \xi}\right)\right| \leqslant \frac{\lambda a+|b(\xi)|}{\lambda a+1} \leqslant 1, \quad|\xi| \leqslant \pi,
$$

so the von Neumann condition holds. By Lemma 2.1(ii) we also have solvability. Hence Theorem 2.2(iii) yields (2.2c); and Theorem 2.1' assures stability.

Example 2.7. Let us keep the boundary conditions in (2.11) with $0<\lambda a<1$, and extend the result of Example 2.6 to nondissipative basic schemes whose characteristic functions satisfy

$$
P(z=-1, \kappa=-1) \neq 0 .
$$

Evidently, we obtain $(2.2 \mathrm{~b}, \mathrm{c})$ precisely as in the previous example. In order to prove (2.2a) we set $\rho=(\lambda a)^{2}$ and observe that $b(\xi)$ in (2.12) satisfies for $0<|\xi|<\pi$,

$$
|b(\xi)|^{4}=\rho^{2}+(1-\rho)^{2}+2 \rho(1-\rho) \cos 2 \xi<\rho^{2}+(1-\rho)^{2}+2 \rho(1-\rho)=1 .
$$

Thus, by (2.12),

or in other words

$$
\left|z\left(\kappa=e^{i \xi}\right)\right| \leqslant \frac{\lambda a+|b(\xi)|}{\lambda a+1}<1, \quad 0<|\xi|<\pi ;
$$

$$
R(z, \kappa) \neq 0, \quad|z| \geqslant 1,|\kappa|=1, \kappa \neq \pm 1
$$

By (2.12) again,

$$
z(\kappa=-1)=-\frac{\lambda a \pm 1}{\lambda a+1}
$$

hence one root is $z(\kappa=-1)=-1$ and the other satisfies $|z(\kappa=-1)|<1$; so

$$
R(z, \kappa=-1) \neq 0, \quad|z| \geqslant 1, z \neq-1 .
$$

Collecting (2.13) $-(2.15)$ we find, therefore, that

$$
\Omega(z, \kappa)=|P(z, \kappa)|+|R(z, \kappa)| \neq 0, \quad|z| \geqslant 1,|\kappa|=1, \kappa \neq 1 .
$$

Thus, (2.2a) is established, and Theorem $2.1^{\prime}$ implies stability.

We remark that certain well-known nondissipative basic schemes satisfy (2.13).

This includes, for example, the unitary Crank-Nicolson scheme

$$
\begin{aligned}
v_{\nu}(t & +\Delta t)-\frac{\lambda a}{4}\left[v_{\nu+1}(t+\Delta t)-v_{\nu-1}(t+\Delta t)\right] \\
& =v_{\nu}(t)+\frac{\lambda a}{4}\left[v_{\nu+1}(t)-v_{\nu-1}(t)\right], \quad \nu=1,2,3, \ldots ;
\end{aligned}
$$

and the almost-dissipative*** backward Euler scheme

$$
\begin{aligned}
v_{\nu}(t+\Delta t)-\frac{\lambda a}{2}\left[v_{\nu+1}(t+\Delta t)-v_{\nu-1}(t+\Delta t)\right]=v_{\nu}(t) & \\
\nu & =1,2,3, \ldots,
\end{aligned}
$$

${ }^{* * *}$ We call a basic scheme almost dissipative if (1.10) holds, except for a finite number of $\kappa,|\kappa|=1$. 
which is included in the family of schemes for which the Gustafsson-KreissSundström theory holds.

If we consider, however, the almost-dissipative Lax-Friedrichs scheme

$$
\begin{aligned}
v_{\nu}(t+\Delta t)=\frac{1}{2}\left[v_{\nu+1}(t)+v_{\nu-1}(t)\right]+\frac{\lambda a}{2}\left[v_{\nu+1}(t)-v_{\nu-1}(t)\right] & \\
0<\lambda a<1, \nu & =1,2,3, \ldots .
\end{aligned}
$$

(which is also admitted by the Gustafsson-Kreiss-Sundström theory; e.g. Osher [10]), we find that $P(z=-1, \kappa=-1)=0$, i.e., (2.13) fails. Indeed, it can be shown that this scheme together with the boundary conditions in (2.11) provides an unstable approximation.

Example 2.8 (compare [15, (3.7)] and the special case [16, Example 6.2]). Take any two-level basic scheme, and determine the boundary conditions by the right-sided, two-step Euler scheme

$$
\begin{aligned}
v_{\nu}(t+\Delta t)=v_{\nu}(t-\Delta t)+2 \lambda a\left[v_{\nu+1}(t-\Delta t)-v_{\nu}(t-\Delta t)\right] \\
0<\lambda a<1 / 2, \nu=0, \ldots, r-1 .
\end{aligned}
$$

Then,

$$
R(z, \kappa)=1-z^{-2}[1+2 \lambda a(\kappa-1)]
$$

so the roots $z(\kappa)$ of $R(z, \kappa)$ satisfy

$$
\left|z\left(\kappa=e^{i \xi}\right)\right|^{4}=1+4 \lambda a(2 \lambda a-1)(1-\cos \xi)<1, \quad 0<|\xi| \leqslant \pi .
$$

That is, our explicit boundary conditions are dissipative and satisfy the von Neumann condition. Hence, by Lemma 2.1(ii) and Theorem 2.2 we obtain (2.2), and Theorem 2.1' assures stability.

Example 2.9 (in [5, Theorem 6.3] and [6, Theorem 2.2]). Consider the unitary, unconditionally stable Crank-Nicolson scheme in (2.16), with $\lambda a \leqslant 1$ and oblique extrapolation at the boundary:

$$
v_{0}(t+\Delta t)=\sum_{j=1}^{\omega}\left(\begin{array}{l}
\omega \\
j
\end{array}\right)(-1)^{j+1} v_{j}[t-(j-1) \Delta t] .
$$

Since we have (2.10), then in order to comply with the assumptions of Theorem 2.1 it remains to show that

$$
\Omega(z, \kappa) \equiv|P(z, \kappa)|+|R(z, \kappa)| \neq 0, \quad z=\kappa,|\kappa|=1, \kappa \neq 1, \lambda a \leqslant 1 ;
$$

so it suffices to prove that

$$
\begin{aligned}
P(z, \kappa) \equiv 1-\frac{\lambda a}{4}\left(\kappa-\kappa^{-1}\right)-z^{-1}\left[1+\frac{\lambda a}{4}\left(\kappa-\kappa^{-1}\right)\right] & \neq 0, \\
z & =\kappa=e^{i \xi}, 0<|\xi| \leqslant \pi, \lambda a \leqslant 1 .
\end{aligned}
$$

Indeed,

$$
P\left(z=e^{i \xi}, \kappa=e^{i \xi}\right)=\left(1-e^{-i \xi}\right)\left[1-\frac{\lambda a}{2}(1+\cos \xi)\right]
$$

so we immediately obtain (2.19), and stability follows.

Example 2.10 (in [6, Theorem 2.2]). Take the almost-dissipative, unconditionally stable backward Euler scheme in (2.17) with the oblique boundary extrapolation in 
(2.18). As in the previous example, in order to prove stability via Theorem 2.1 it is sufficient to show that

$$
P(z, \kappa) \equiv 1-\frac{\lambda a}{2}\left(\kappa-\kappa^{-1}\right)-z^{-1} \neq 0, \quad z=\kappa=e^{i \xi}, 0<|\xi| \leqslant \pi .
$$

Since

$$
P\left(z=e^{i \xi}, \kappa=e^{i \xi}\right)=1-\cos \xi+i(1-\lambda a) \sin \xi,
$$

we get (2.20), and stability is established.

3. Proofs and Remarks. In order to prove Theorems 2.1 and 2.2 we begin by considering the basic characteristic function $P(z, \kappa)$ in (1.7). By Assumption 1.4, for each $z$ with $|z| \geqslant 1, P(z, \kappa)$ has $r+p$ roots $\kappa(z)$. These roots, which play a major role in the stability analysis of approximation (1.6), have the following separation property:

LemMA 3.1 ([3, Lemma 4.2]; compare [5, Lemmas 5.1 and 5.2]). For $|z|>1$, the basic characteristic function $P(z, \kappa)$ has precisely $r$ roots $\kappa(z)$ with $0<|\kappa(z)|<1, p$ roots with $|\kappa(z)|>1$, and no roots with $|\kappa(z)|=1$.

According to this lemma, the roots $\kappa(z)$ of $P(z, \kappa)$ split for $|z|>1$ into two groups: $r$ inner roots satisfying $|\kappa(z)|<1$, and $p$ outer roots with $|\kappa(z)|>1$. Using Assumption 1.4 and a continuity argument, we see that these groups of inner and outer roots remain well defined for $|z| \geqslant 1$ as well, where milder inequalities, $|\kappa(z)| \leqslant 1$ and $|\kappa(z)| \geqslant 1$, hold, respectively. Since by Assumption $1.4, \kappa=0$ is not a root of $P(z, \kappa)$ for $|z| \geqslant 1$, we obtain

Lemma 3.2 ([3, Lemma 4.3]). For $|z| \geqslant 1$, the $r+p$ roots $\kappa(z)$ of the basic characteristic function $P(z, \kappa)$ split into $r$ inner roots with $0<|\kappa(z)| \leqslant 1$, and p outer roots with $|\kappa(z)| \geqslant 1$.

We can now quote our main preliminary stability criterion in [3]:

THEOREM 3.1 ([3, Theorem 4.2]). Approximation (1.6) is stable if and only if for every $z$ with $|z| \geqslant 1$ and each corresponding inner root $\kappa(z)$ of $P(z, \kappa)$, the boundary characteristic function $R(z, \kappa)$ satisfies

$$
R(z, \kappa(z)) \neq 0 .
$$

If $\kappa(z)$ is an inner root for $z,|z| \geqslant 1$, then by definition,

$$
P(z, \kappa(z))=0 \text {. }
$$

Thus, combining this fact with (3.1), we immediately obtain

COROllary 3.1. Approximation (1.6) is stable if and only if for every $z$ with $|z| \geqslant 1$ and each corresponding inner root $\kappa(z)$ of $P(z, \kappa)$,

$$
\Omega(z, \kappa(z)) \equiv|P(z, \kappa(z))|+|R(z, \kappa(z))| \neq 0 .
$$

We shall need yet another result which describes the behavior of the inner roots for $z=1$ :

Lemma 3.3 ([3, Lemma 5.1]). If $z=1$, then $\kappa=1$ is not an inner root of $P(z, \kappa)$.

This brings us to

Proof of Theorem 2.1. Take any $z,|z| \geqslant 1$, and let $\kappa(z)$ be a corresponding inner root of $P(z, \kappa)$. If $z \neq 1$, then by Lemma 3.2 , we have $0<|\kappa(z)| \leqslant 1$; so (2.1) 
implies (3.2). If on the other hand, $z=1$, then by Lemma 3.3, $\kappa(z)=1$ is excluded as an inner root; so by Lemma 3.2 we have $0<|\kappa(z)| \leqslant 1, \kappa(z) \neq 1$, and again (2.1) yields (3.2). By Corollary 3.1, therefore, approximation (1.6) is stable, and the proof is complete.

Proof of Theorem 2.2. (i) If either the basic scheme or the boundary conditions are dissipative, then by Assumption 1.3(i) and Definition 2.1, either

$$
P(z, \kappa) \neq 0, \quad|z| \geqslant 1,|\kappa|=1, \kappa \neq 1,
$$

or

$$
R(z, \kappa) \neq 0, \quad|z| \geqslant 1,|\kappa|=1, \kappa \neq 1
$$

and (2.2a) follows.

(iia) Since the basic scheme is consistent with (1.5), it is at least first-order accurate; so, in particular, it satisfies the ordinary zero-order accuracy condition

$$
\sum_{j=-r}^{p} a_{j(-1)}=\sum_{\sigma=0}^{s} \sum_{j=-r}^{p} a_{j \sigma}
$$

which we equivalently write as

$$
P(z=1, \kappa=1)=0 .
$$

By assumption, the basic scheme is two-level, so the function $P(z, \kappa=1)$ is a first degree polynomial in $z^{-1}$ which, by (3.3), has a unique root $z^{-1}=1$. Thus,

$$
P(z, \kappa=1) \neq 0, \quad|z| \geqslant 1, z \neq 1,
$$

and we obtain $(2.2 b)$.

(iib) In the 3-level case we still have (3.3), but now $P(z, \kappa=1)$ is a second degree polynomial in $z^{-1}$ with real coefficients. By (3.3), $z^{-1}=1$ is one of the roots, so the other root must be real as well; thus

$$
P(z, \kappa=1) \neq 0, \quad|z|=1, z \neq \pm 1 .
$$

In addition, since the basic scheme satisfies the von Neumann condition (Assumption 1.2(i)), then

$$
P(z, \kappa=1) \neq 0, \quad|z|>1 .
$$

By (3.4) and (3.5) therefore,

$$
\Omega(z, \kappa=1) \neq 0, \quad|z| \geqslant 1, z \neq \pm 1,
$$

which together with $(2.5)$ gives $(2.2 b)$.

The proof of (iic) is identical to that of (iia), except that $P(z, \kappa)$ is replaced by $R(z, \kappa)$, where by the zero-order accuracy of the boundary conditions, we have $R(z=1, \kappa=1)=0$ instead of (3.3).

For the proof of (iid) we still have (3.5); and since the boundary conditions are three-level we may replace (3.4) by

$$
R(z, \kappa=1) \neq 0, \quad|z|=1, z \neq \pm 1 .
$$

By (3.5) and (3.7) we obtain (3.6) again, which together with (2.5) yields (2.2b).

(iii) Since the boundary conditions are assumed to be boundedly solvable, then by Lemma 2.1(i) we have

$$
T_{-1}(\kappa) \equiv \sum_{j=0}^{q} c_{j(-1)} \kappa^{j} \neq 0, \quad 0<|\kappa| \leqslant 1 .
$$


Moreover, by (1.6c) and (2.6),

$$
T_{-1}(0)=c_{0(-1)} \neq 0 .
$$

So, combining $(3.8 \mathrm{a}, \mathrm{b})$ we obtain

$$
T_{-1}(\kappa) \neq 0, \quad|\kappa| \leqslant 1 .
$$

Now, the function $z^{q+1} R(z, \kappa)$ is a polynomial in $z$ and $\kappa$, so it can be written as

$$
z^{q+1} R(z, \kappa)=\left(z-z_{1}\right) \cdots\left(z-z_{d}\right) R_{0}(z, \kappa),
$$

where $R_{0}(z, \kappa)$ is a polynomial in $\kappa$ whose leading coefficient is a nonvanishing constant and whose other coefficients are rational functions of $z$. By Assumption 1.5, the boundary characteristic function

$$
R(z, \kappa) \equiv \sum_{j=0}^{m} c_{j}(z) \kappa^{j}
$$

is not the zero polynomial in $\kappa$ for any (fixed) $z$ with $|z| \geqslant 1$. Thus, the $z_{j}$ in (3.10) must satisfy

$$
\left|z_{j}\right|<1, \quad j=1, \ldots, d .
$$

It follows that the leading coefficient of $z^{q+1} R(z, \kappa)$ (as a polynomial in $\kappa$ ) is uniformly bounded away from zero for all $|z| \geqslant 1$. Hence, the roots $\kappa=\kappa(z)$ of $z^{q+1} R(z, \kappa)$, and therefore those of $R(z, \kappa)$, are continuous functions of $z$ for $|z| \geqslant 1$.

Since by hypothesis, the boundary conditions satisfy the von Neumann condition, then for $|z|>1$, the function $R(z, \kappa)$ does not vanish on the unit circle $|\kappa|=1$. Hence, by the continuity established above, the number of roots $\kappa=\kappa(z)$ of $R(z, \kappa)$ satisfying $|\kappa(z)| \leqslant 1$, is independent of $z,|z|>1$, and it thus equals the number of roots $\kappa,|\kappa| \leqslant 1$, of the function

$$
R(z \rightarrow \infty, \kappa) \equiv T_{-1}(\kappa)
$$

By (3.9) therefore, $R(z, \kappa)$ has no roots $|\kappa| \leqslant 1$ for $|z|>1$, so the roots $\kappa(z)$ of $R(z, \kappa)$ satisfy $|\kappa(z)|>1$ if $|z|>1$. Thus, for $|z| \geqslant 1$, these continuous roots satisfy $|\kappa(z)| \geqslant 1$, i.e.,

$$
R(z, \kappa) \neq 0, \quad|\kappa|<1,|z| \geqslant 1,
$$

and $(2.2 \mathrm{c})$ follows.

In concluding the paper we make the following remarks.

Remark 3.1. Our main results in [3] follow immediately from the present ones. Indeed, consider Theorem 3.3 of [3] where we assume that the basic scheme is dissipative, that the boundary conditions are boundedly solvable and satisfy the von Neumann condition, and that

$$
R(z, \kappa=1) \neq 0, \quad|z|=1, z \neq 1 .
$$

With these assumptions, Theorem 2.2(i)(iii) gives (2.2a, c). Moreover, by Assumption 1.2(i) we have (3.5), which together with (3.12) yields (2.2b). Thus, Theorem $2.1^{\prime}$ implies stability, and Theorem 3.3 of [3] follows. 
In Theorem 3.4 of [3] it is assumed that the basic scheme is arbitrary, that the boundary conditions are boundedly solvable and dissipative, and that (3.11) holds. Again, Theorem 2.2(i)(iii) yields (2.2a, c), and (3.11) together with (3.5) gives (2.2b); so Theorem 2.1' implies Theorem 3.4 of [3].

Theorems 3.1 and 3.2 in [3] follow from Theorem 2.1' and 2.2 with similar ease.

Remark 3.2. Examples 2.1-2.6 contain all the main examples in [3]. Example 2.3 with a nondissipative basic scheme, and Examples 2.7-2.10, are new in the sense that they could not have been handled by our old results in [3]. For Examples 2.3, 2.7, 2.9 and 2.10 this is true since Theorems 3.1-3.4 in [3] require that either the basic scheme or the boundary conditions be dissipative. For Example 2.8, where the basic scheme is not necessarily dissipative but the boundary conditions are, one may hope that Theorem 3.4 of [3] would help. But $R(z=-1, \kappa=1)=0$; so (3.7) in [3] is violated, and Theorem 3.4 of [3] fails.

Remark 3.3. Not all interesting cases are covered by our results. For instance, Gustafsson et al. showed in Theorem 6.1 of [5] that the Leap-Frog scheme in (2.8) together with the linear oblique boundary extrapolation

$$
v_{0}(t+\Delta t)=2 v_{1}(t)-v_{2}(t-\Delta t)
$$

provides a stable approximation. We easily find, however, that

$$
\Omega(z=-1, \kappa=-1)=|P(z=-1, \kappa=-1)|+|R(z=-1, \kappa=-1)|=0,
$$

so (2.1) may not hold. Consequently, Theorem 2.1 fails for this example, showing that our criteria are sufficient but not necessary for stability.

Remark 3.4. Let us show that Assumption 1.5 is necessary for the stability of approximation (1.6). Indeed, if Assumption 1.5 fails, then at some $z=z_{0}$ with $\left|z_{0}\right| \geqslant 1$, all the $c_{j}(z)$ in (1.12) vanish simultaneously. Hence,

$$
R\left(z=z_{0}, \kappa\right) \equiv \sum_{j=0}^{m} c_{j}\left(z_{0}\right) \kappa^{j} \equiv 0 ;
$$

that is, $R\left(z=z_{0}, \kappa\right)$ is the zero polynomial in $\kappa$. By Lemma 3.2, for $z=z_{0}$, the basic characteristic function $P(z, \kappa)$ has $r$ inner roots $\kappa\left(z_{0}\right)$ with $0<\left|\kappa\left(z_{0}\right)\right| \leqslant 1$. For each of these roots (3.12) implies

$$
R\left(z=z_{0}, \kappa=\kappa\left(z_{0}\right)\right)=0,
$$

and Theorem 3.1 assures instability.

For example, consider any dissipative basic scheme with boundary conditions determined by the two-level, zero-order vertical extrapolation:

$$
v_{\nu}(t+\Delta t)=v_{\nu}(t), \quad \nu=0, \ldots, r-1 .
$$

Clearly, these explicit boundary conditions are boundedly solvable (Lemma 2.1), and they satisfy the von Neumann condition. Thus, the hypotheses of Theorem 2.2 hold, and Theorem 2.1' seems to imply stability. As mentioned in Section 4 of [14], however, the approximation is unstable. The reason for this instability is that the boundary conditions in (3.13) fail to satisfy Assumption 1.5, since

$$
\sum_{j=0}^{m}\left|c_{j}(z)\right|_{z=1}=\left|1-z^{-1}\right|_{z=1}=0 \text {. }
$$


This example, though found unstable in the sense of Definition 3.3 of [5], is stable in the $l_{2}$ sense described in [14].

Remark 3.5. It is known that the accuracy of the boundary conditions plays a decisive role in determining the overall rate of convergence of the difference approximation. In fact, Gustafsson [4] has shown that if approximation (1.6) is stable and the basic scheme is accurate of order $\delta$, then an overall $\delta$ rate of convergence is assured if the boundary conditions are of $\delta-1$ order of accuracy at least.

Now, if we use horizontal or oblique boundary extrapolation as in (2.7) or (2.9), then obviously there is no restriction on accuracy at the boundary. In general, however, the question of maximal possible accuracy for the boundary conditions can be quite difficult if the boundary conditions are to satisfy certain properties. For instance, it is not hard to see that most of the boundary conditions in the examples of Section 2 are stable in the sense of the definition in Assumption 1.2, with $P(z, \kappa)$ replaced by the characteristic boundary function $R(z, \kappa)$. Thus, it seems natural to raise the question of maximal accuracy for stable boundary conditions of type (1.6c). This question was addressed by Iserles and Strang [7, Theorems 1 and 2] who showed that stable, two-level boundary conditions of type (1.6c) may not be more than second-order accurate. On the other hand multilevel, stable boundary conditions can be constructed to any order of accuracy if $q$ in (1.6c) is chosen sufficiently large.

Department of Mathematics

Technion-Israel Institute of Technology

Haifa 32000, Israel

and

Institute for the Interdisciplinary

Applications of Algebra and Combinatorics

University of California

Santa Barbara, California 93106

Department of Applied Mathematics

School of Mathematical Sciences

Tel Aviv University

Ramat-Aviv 69978, Israel

and

Institute for Computer Applications

in Science and Engineering

NASA Langley Research Center

Hampton, Virginia 23665

1. M. Goldberg, "On a boundary extrapolation theorem by Kreiss," Math. Comp., v. 31, 1977, pp. 469-477.

2. M. Goldberg \& E. TADMOR, "Scheme-independent stability criteria for difference approximations of hyperbolic initial-boundary value problems. I," Math. Comp., v. 32, 1978, pp. 1097-1107.

3. M. Goldberg \& E. TADMOR, "Scheme-independent stability criteria for difference approximations of hyperbolic initial-boundary value problems. II," Math. Comp., v. 36, 1981, pp. 603-626.

4. B. GuSTAFSSON, "The convergence rate for difference approximations to mixed initial boundary value problems," Math. Comp., v. 29, 1975, pp. 396-406.

5. B. Gustarsson, H.-O. KREISS \& A. SUNDSTROM, "Stability theory of difference approximations for mixed initial-boundary value problems. II," Math. Comp., v. 26, 1972, pp. 649-686.

6. B. GUSTAFSSON \& J. Oliger, "Stable boundary approximations for implicit time discretizations for gas dynamics,” SIAM J. Sci. Statist. Comput., v. 3, 1982, pp. 408-421. 
7. A. Iserles \& G. Strang, “The optimal accuracy of difference schemes," Trans. Amer. Math. Soc., v. 277,1983 , pp. $779-803$.

8. H.-O. KREISS, "Difference approximations for hyperbolic difference equations," in Numerical Solutions of Partial Differential Equations (J. H. Bramble, ed.), Academic Press, New York, 1966, pp. $51-58$

9. H.-O. Kreiss \& J. Oliger, Methods for the Approximate Solution of Time Dependent Problems, GARP Publication Series No. 10, Geneva, 1973.

10. S. OSHER, "Systems of difference equations with general homogeneous boundary conditions," Trans. Amer. Math. Soc., v. 137, 1969, pp. 177-201.

11. S. OSHER, "Stability of parabolic difference approximations to certain mixed initial-boundary value problems," Math. Comp., v. 26, 1972, pp. 13-39.

12. G. SkOllermo, How the Boundary Conditions Affect the Stability and Accuracy of Some Implicit Methods for Hyperbolic Equations, Report No. 62, Dept. of Computer Science, Uppsala University, Uppsala, Sweden, 1975.

13. G. SKOllermo, Error Analysis for the Mixed Initial Boundary Value Problem for Hyperbolic Equations, Report No. 63, Dept. of Computer Science, Uppsala University, Uppsala, Sweden, 1975.

14. E. TADMOR, "The unconditional instability of inflow-dependent boundary conditions in difference approximations to hyperbolic systems," Math. Comp., v. 41, 1983, pp. 309-319.

15. E. TADMOR, Scheme-Independent Stability Criteria for Difference Approximations to Hyperbolic Initial-Boundary Value Systems, Ph. D. Thesis, Department of Mathematical Sciences, Tel Aviv University, Tel Aviv, Israel, 1978.

16. L. N. Trefethen, Wave Propagation and Stability for Finite Difference Schemes, Ph. D. Thesis, Report No. STAN-CS-82-905, Computer Science Department, Stanford University, Stanford, California, 1982. 